ISSN 2075-4698

www.mdpi.com/journal/societies

Article

\title{
Peer Attachment and Cyber Aggression Involvement among Chinese, Indian, and Japanese Adolescents
}

\section{Michelle F. Wright ${ }^{1, *}$, Ikuko Aoyama ${ }^{2}$, Shanmukh V. Kamble ${ }^{3}$, Zheng Li ${ }^{4}$, Shruti Soudi ${ }^{3}$, Li Lei ${ }^{4}$ and Chang Shu ${ }^{4}$}

1 Faculty of Social Studies, Masaryk University, Brno 60200, Czech Republic

2 Office for the Promotion of Global Education Programs, Shizuoka University, Shizuoka Prefecture 432-8561, Japan; E-Mail: aoyama.ikuko@shizuoka.ac.jp

3 Department of Psychology, Karnatak University, Karnataka State 580 003, India; E-Mails: skamble@kud.ac.in (S.V.K.); ssoudi6@gmail.com (S.S.)

4 Department of Psychology, Renmin University of China, Beijing 100872, China; E-Mails: lizheng0723@ruc.edu.cn (Z.L.); lei.li@ruc.edu.cn (L.L.); krystal199009@ruc.edu.cn (C.S.)

* Author to whom correspondence should be addressed; E-Mail: michelle.wright@mail.muni.cz; Tel.: +420-549-49-5259.

Academic Editors: Conor Mc Guckin and Lucie Corcoran

Received: 30 December 2014 / Accepted: 13 April 2015 / Published: 22 April 2015

\begin{abstract}
Significant advancements have been made in cyber aggression literature, with many studies revealing the consequences associated with adolescents' involvement in these behaviors. Few studies have focused on cyber aggression involvement in China, India, and Japan. The present study examined differences in cyber aggression perpetration and victimization among 1637 adolescents living in China, India, and Japan, while controlling for face-to-face bullying involvement, individualism, and collectivism. Another aim of the present study was to examine country of origin and cyber aggression involvement (i.e., the uninvolved, cyberaggressor-cybervictims, cyberaggressors, and cybervictims) differences in peer attachment. Findings revealed that adolescents from India had the highest levels of cyber aggression involvement when compared to adolescents from China or Japan. Chinese adolescents engaged in more cyber aggression perpetration and were victimized more by cyber aggression when compared to Japanese adolescents. No country of origin differences were found for peer attachment. However, uninvolved adolescents reported higher levels of peer attachment when compared to the other groups. Cyberaggressorcybervictims had the lowest levels of peer attachment, followed by cybervictims and
\end{abstract}


cyberaggressors. These results suggest that there should be concern about cyber aggression involvement among adolescents in these countries, especially in India, where cyber aggression research has been slow to develop.

Keywords: cyberbullying; cyber aggression; cyber victimization; peer attachment; individualism; collectivism; culture; China; India; Japan; adolescents

\section{Introduction}

Most adolescents have spent their lives completely enmeshed in a digital world, with various opportunities and information at their fingertips. Technology usage has many benefits for adolescents, allowing them to quickly communicate with friends and family and to access a wealth of information quickly. Despite such benefits, adolescents also experience risks associated with their technology usage. Cyber aggression is one risk, and it has received attention from researchers, educators, parents, and the general public. Research on cyber aggression is increasing, but research focused on these behaviors in other countries has been slower to develop, particularly in China, India, and Japan. Although the literature has been advancing on cyber aggression, moving from frequency rates to the behavioral characteristics and consequences, little attention has been given to the role of peer relationships in these behaviors. Of this literature, research indicates that perpetrators of cyber aggression are often peers at adolescents' schools and that peer rejection is related to cyber aggression, perpetration, and victimization [1,2]. Furthermore, poor peer attachment is related positively to cyber aggression involvement. However, little attention has been given to examining country of origin and cyber aggression involvement differences in peer attachment. To address this gap in the literature, the present study examined differences in cyber aggression perpetration and victimization among Chinese, Indian, and Japanese adolescents as well as the roles of country of origin and cyber aggression involvement in adolescents' perceptions of their peer attachment.

\section{Cyber Aggression Involvement and Culture}

This study utilizes the terminology of cyber aggression, which is a broader form of cyberbullying. Cyber aggression includes intentionally harmful behaviors, such as hacking someone's online accounts, sending degrading messages, spreading rumors, and calling others mean names [3]. These behaviors are directed toward others who find such behaviors offensive and unwanted, and such behaviors can occur through email, chat programs, text messages, gaming consoles, social networking sites, blogs, and discussion boards. Unlike cyberbullying, Grigg [3] argues that aggressive cyber behaviors do not always include repetition. This component is central to the traditional bullying and cyberbullying definitions. Therefore, measures of cyberbullying focus on repetition while cyber aggression measures do not include the repetition component. This literature review uses both terminologies in order to accurately describe the terminology and methodology of the studies. In addition, cyber aggressive behaviors also include those behaviors that do not have a face-to-face equivalent, like hacking someone's Facebook account. Hacking, as a form of cyber aggression, is carried out with malicious intent, with the desire to damage someone's reputation and/or their relationships. 
Extensive research has focused on the factors which predict adolescents' involvement in cyber aggression and cyberbullying. In this literature, face-to-face aggression, face-to-face victimization, and cyber victimization are all found to be associated positively with cyber aggression and cyberbullying perpetration [2,4-8]. Other research implicates peer rejection, a lack of empathy, beliefs about anonymity, and narcissism as predictors of cyber aggression and cyberbullying perpetration [2,9-11]. Research aimed at understanding cyber aggression and cyberbullying involvement is important, as these behaviors relate to adjustment difficulties, specifically depression, anxiety, and loneliness [12-14]. In addition, cyber aggression and cyberbullying involvement is linked to poor academic performance, increased absences, and more truancy [1,15-17]. Researchers have classified aggression involvement into different categories including the uninvolved (neither perpetrator nor victim), cybervictims, cyberbullies, and cyberbully-cybervictims (both perpetrator and victim) [13].

Cyber aggression and cyberbullying research is even more important as research indicates that this phenomenon is not only found in one country, though much of the research has been conducted in the United States. The available research suggests that cyber aggression is a global concern. Of these studies, researchers have identified cyber aggression and cyberbullying involvement in Australia [12], Belgian [18], Germany [19], Ireland [20], Italy [21], Spain [22], Sweden [23,24], and Turkey [25].

Research on cyber aggression and cyberbullying involvement has been slower to develop in Asia, with findings revealing that perpetration and victimization occurs in some countries and areas, including China [26], Korea [27], Singapore [28], and Taiwan [16]. Examining cyber aggression involvement in Asia is imperative as the top four countries according to internet usage include China, India, and Japan, as well as the United States [29]. China ranks as number one, followed by the United States, India, and Japan. Understanding where a country ranks in terms of internet usage is important as access to the internet and the more time spent online are both risk factors associated with cyberbullying perpetration and victimization [30,31].

Given the high levels of internet consumption in China, India, and Japan, more research attention should focus on adolescents' involvement in cyber aggression in these countries. Although some research has examined cyberbullying involvement in China, this research has focused on frequency rates, demographic variables, and lower academic achievement as factors linked to the perpetration of these behaviors [26,32]. Few empirical investigations exist concerning cyberbullying perpetration and victimization in India and Japan. In one study, Japanese adolescents reported cyberbullying, but their levels of involvement were lower than adolescents from the United States and Austria [33,34]. Studies conducted in India focus on cyber gender harassment, a form of cyber harassment involving similar behaviors as cyberbullying, except that this behavior occurs among adults instead of children and adolescents [35]. Taken together, research from China, India, and Japan indicate that cyber aggression and cyberbullying occur among adolescents in these countries, and that their high levels of internet consumption warrant further investigation.

Furthermore, cultural values, including collectivism and individualism, impact adolescents' involvement in aggressive behaviors [36-39]. Collectivistic countries, like China and Japan, promote, prime, and reinforce individuals for behaving consistently with an interdependent self-construal [40]. Individualistic countries, like the United States, reinforce independent self-construal. India is considered both a collectivistic and an individualistic country, which might place these adolescents at an elevated risk of cyber aggression involvement when compared to adolescents from China and Japan. In the literature, collectivism is related negatively to aggression involvement, while 
individualism is associated positively with aggression perpetration and victimization [34,40]. Few studies have examined whether cultural values influence adolescents' perpetration and victimization by cyber aggression. Barlett and colleagues [33] examined interdependent self-construal as a moderator in the relationship between country of origin (i.e., United States, Japan) and cyberbullying perpetration. The results revealed that cyberbullying was highest for young adults from the United States when they endorsed low levels of interdependent self-construal. These results were not found for Japanese young adults. However, it isn't clear whether independent self-construal would impact cyber aggression perpetration. Based on the previous research on face-to-face aggression perpetration, it might be likely that independent self-construal increases the risk of engaging in cyber aggression $[34,40]$.

Gender differences in cyber aggression involvement in the United States and in European countries are mixed [2,9,41-43]. The literature on gender differences in cyber aggression perpetration and victimization is not as mixed in Asian countries. In this research, Chinese boys perpetrate and are victimized by cyberbullying more often than Chinese girls $[26,32,44]$. Similar patterns were found in Japan as well, with Japanese young adult males perpetrating these behaviors at higher rates than Japanese young adult females [33]. It is unclear whether Japanese males would experience more or less victimization than Japanese females as there has been no research conducted on this topic. In addition, research has not been conducted on gender differences in cyber aggression involvement among Indian adolescents. Given that research on cyber gender harassment conducted in India focuses solely on men harassing women through digital technologies, it might be likely that girls are more at risk for cyber victimization while boys might perpetrate cyber aggression more often than girls [35].

\section{Peer Attachment and Aggression}

High peer attachment involves adolescents' internationalization of the knowledge that their peers will be available and responsive when needed [36]. Problems within peer relationships can place adolescents at a higher risk of being involved in conflicts with their peers [37]. Thus, it is not surprising that adolescents with higher rates of victimization and those with behavioral problems are likely to rate their peer relationships as poor, due to these adolescents being less socially integrated in the peer group [45,46]. These adolescents also show less empathy toward their peers and this lack of empathy combined with behavioral problems might exacerbate their aggression directed toward their peers. Furthermore, social integration is directly related to adolescents' peer relationships, and when such integration is low, these adolescents are unable to manage and deal with relationships effectively [46]. When ostracized by the peer group, adolescents often act reactively by using aggression, developing favorable attitudes toward these behaviors [47]. In the literature, higher peer attachment relates to more sympathetic attitudes toward peers, and less delinquency and aggressive behaviors [48]. Peer attachment is also related negatively to face-to-face bullying and cyberbullying as well as victimization by both types of bullying. Some attention has focused on the relationship between peer attachment and cyberbullying categories. One of the few studies conducted on these associations found that cyberbullycybervictims have lower levels of peer attachment than uninvolved adolescents [49]. No other research exists concerning differences in peer attachment among cyberbullies and cybervictims. Gender has also been investigated as a factor relating to peer attachment. Research suggests that girls are more attached to their peers than boys, though it isn't clear how cyber aggression classification type might alter these associations [50]. 
Little attention has been given to examining peer attachment among adolescents in China, India, and Japan. The literature suggests that members of collectivistic cultures are attached to their peers, due to the reinforcement of interdependence and maintaining relationships with others in their society [51]. Therefore, considering that countries, like China, India, and Japan, have collectivistic focuses, it is likely that these adolescents are also attached to their peers. Given that individualism is endorsed in India as well, these adolescents might experience different levels of peer attachment than adolescents from China and Japan. In the literature, adolescents from the United States had higher levels of peer attachment than adolescents from India [52]. On the other hand, other research indicates that Chinese adolescents who immigrated to Italy have higher rates of peer attachment than Italian adolescents [53]. Considering these contrasting findings, it is difficult to conclude whether Indian adolescents experience higher or lower levels of peer attachment when compared to adolescents from China and India. In one of the few studies to investigate peer attachment in relation to aggression, Yang and colleagues [54] found that poor peer attachment related positively to Chinese adolescents' aggression and delinquency. These patterns are similar to those found in the United States, though it isn't clear whether similar patterns would be found in India and Japan. In addition, there is no literature revealing the role of cyber aggression categories (i.e., the uninvolved, cybervictims, cyberaggressors, cyberaggressors-cybervictims) and country of origin in adolescents' attachment to their peers.

\section{Present Study}

Few investigations have been conducted on cyber aggression involvement among adolescents in China, India, and Japan, especially studies conducted to compare rates across these countries. In addition, it is unknown whether peer attachment relates to cyber aggression perpetration and victimization. To this end, the first aim of the present study compared rates of cyber aggression involvement in China, India, and Japan, while controlling for face-to-face bullying perpetration and victimization, individualism, and collectivism. It was hypothesized that Indian adolescents would report higher levels of cyber aggression involvement when compared to Chinese and Japanese adolescents, given the emphasis on both collectivism and individualism in India [35]. Although China and Japan are both collectivistic societies, China is a little less collectivistic and has higher rates of internet consumption, which might contribute to adolescents in this country being more at risk for cyber aggression involvement [29]. Therefore, it was hypothesized that Chinese adolescents would report higher levels of cyber aggression involvement than Japanese adolescents. Another aim was to examine the role of gender in adolescents' cyber aggression perpetration and victimization based on country of origin. Chinese and Japanese boys were expected to engage in more cyber aggression perpetrators than girls from these countries. In addition, Chinese boys were also expected to experience more cyber victimization when compared to Chinese girls. Due to the research on cyber gender harassment in India, it was expected the girls would experience more cyber victimization, whereas boys would be more likely to be the perpetrators of cyber aggression [35].

The second aim of the present study was to examine differences in cyber aggression involvement categories (i.e., the uninvolved, cybervictims, cyberaggressors, cyberaggressors-cybervictims) for peer attachment among Chinese, Indian, and Japanese adolescents. Therefore, three-way interactions were examined among gender, country of origin, and cyber aggression involvement. Since Indian adolescents were hypothesized to have higher levels of cyber aggression involvement, it was also hypothesized that 
cyberaggressors-cybervictims from India would have the lowest levels of peer attachment when compared to cyberaggressors-cybervictims from China and Japan. Uninvolved adolescents were expected to have the highest peer attachment, despite their country of origin. No other hypotheses were made regarding the interaction of cyber aggression involvement classifications and gender.

\section{Methods}

\subsection{Participants}

Participants were 1637 adolescents (age range $11-15$ years old; $48.3 \%$ girls) from China ( $n=683$; $46.7 \%$ girls), India ( $n=480 ; 46.5 \%$ girls), and Japan $(n=474 ; 52.6 \%$ girls $)$. In China, data was collected from two schools, with one located in Beijing and the other in the An Hui Province. Adolescents from India were from six schools in the Karnataka state of India. Japanese adolescents were recruited from two schools located in a suburb of Tokyo. All data was collected in the Fall of 2013, except for Japanese adolescents. Japanese schools begin in April, and data was collected in July 2014.

\subsection{Procedures and Measures}

Emails were sent to principals from target schools, describing the purpose of the study, how the school could participate, and what adolescents would be expected to do. When principals expressed an interest in the study, a meeting was setup with principals and teachers in order to receive their permission for their students to participate in the study. All principals and teachers agreed to allow students to participant in the study. Consent documents were sent home with adolescents, and then returned to their teachers, except in Japan where consent was obtained from school principals only. On the day of data collection, adolescents provided their assent to participate in the study before completing the surveys. No adolescents refused to participate. This study is part of a larger study on the psychosocial development of adolescents from various countries around the world, with a major focus on understanding the contextual factors which influence their involvement in cyber aggression. For this study, the following questionnaires were administered, including individualism and collectivism, face-to-face aggression involvement, cyber aggression involvement, and peer attachment. All consent, assent, and questionnaires were translated into the primary language of adolescents' country of origin, and then back-translated by researchers fluent in both English and the language of the country of origin.

\subsubsection{Individualism and Collectivism}

This questionnaire assessed adolescents' endorsement of individualism and collectivism [55]. $\mathrm{Li}$ and colleagues [55] adapted the Horizontal and Vertical Individualism and Collectivism measure [56] by changing some items to be suitable for adolescents (e.g., "It is important that I do my work better than others" was changed to "It is important that I do my schoolwork better than others"). There were sixteen items included in this measure, with eight for individualism (e.g., Winning is everything) and eight for collectivism (e.g., Family members should stick together, no matter what sacrifices are required). Participants rated the items on a scale of 1 (Absolutely disagree) to 9 (Absolutely agree). Both subscales demonstrated adequate reliability ( $\alpha=0.92$ for individualism; $\alpha=0.79$ for collectivism). 


\subsubsection{Face-to-Face Aggression Involvement}

To examine face-to-face aggression involvement, adolescents completed a questionnaire concerning how often they perpetrated face-to-face aggression (e.g., How often do you tell a peer that you will not like him or her unless he or she does what you want?) and were victimized by face-to-face aggression (e.g., How often does a peer say they won't like you unless you do what he or she wants you to do?) [57]. The items were described as occurring within the current school year. Adolescents rated the eighteen items (nine per subscale) on a scale of 1 (Never) to 5 (All of the Time). Face-to-face aggression perpetration had a Cronbach's alpha of 0.79 and 0.81 for face-to-face victimization.

\subsubsection{Cyber Aggression Involvement}

Adolescents indicated how often they perpetrated cyber aggression (e.g., How often do you spread bad rumors about another peer online or through text messages?) and were victimized by cyber aggression (e.g., How often does a peer spread bad rumors about you online or through text messages?) [11]. Eighteen items were included on this measure, with nine items per subscale. The items were described as occurring within the current school year. They rated all items on a scale of 1 (Never) to 5 (All of the Time). Cronbach's alphas were acceptable for both cyber aggression perpetration $(\alpha=0.90)$ and cyber aggression involvement $(\alpha=0.90)$.

\subsubsection{Peer Attachment}

The peer attachment subscale of the Inventory of Parent and Peer attachment was used to assess adolescents' perceptions of the positive and negative dimensions of their relationship with their peers [58]. There were 25 items used, each rated on a scale of 1 (Almost Never or Never True) to 5 (Almost Always or Always True). Cronbach's alpha was 0.89 for this measure.

\section{Results}

To examine the hypotheses for this study, two separate sets of analyses were performed. The first analysis examined differences among adolescents from the three countries regarding their cyber aggression perpetration and victimization. The second analysis investigated the role of cyber aggression involvement in peer attachment, and the differences across the three countries. Bonferroni corrections were utilized for all post-hoc follow-up analyses. Multi-group factor analysis was performed in Mplus for all four measures. Measurement invariance was not found among any of the groups. In addition, the MANOVAs and ANOVAs were performed without the cultural values, but the models with cultural values were better. Therefore, the models included cultural values. Interested readers should contact the first author for more information about these additional analyses.

\subsection{Differences in Cyber Aggression Involvement}

A MANOVA was conducted with cyber aggression perpetration and victimization as the dependent variables, country and gender as the independent variables, and face-to-face aggression involvement (perpetration and victimization) and cultural values (individualism and collectivism) as covariates. An interaction was included between country and gender. Main effects of country (Wilks' $\Lambda=0.85$, 
$F(4,3130)=65.83, p<0.001)$ and gender (Wilks' $\Lambda=0.97, F(2,1565)=11.34, p<0.001)$ were found. The interaction was also significant (Wilks' $\Lambda=0.99, F(4,3130)=4.75, p<0.001$ ).

Next, follow-up ANOVAs for cyber aggression perpetration and victimization were conducted with the same variables used in the MANOVA (see Table 1 for correlations and Table 2 for means and standard deviations). Similar main effects were found for cyber aggression perpetration (country: $F(2,1565)=129.21, p<0.001$; gender: $F(1,1565)=21.55, p<0.001)$ and cyber aggression victimization (country: $F(2,1565)=56.72, p<0.001$; gender: $F(1,1565)=15.07, p<0.001$ ). Interactions were also significant for cyber aggression involvement (perpetration: $F(2,1565)=8.70$, $p<0.001$; victimization: $F(2,1565)=6.41, p<0.01$ ). Indian adolescents (perpetration: $M=1.86$; $S D=0.74$; victimization: $M=1.79 ; S D=0.86$ ) reported greater cyber aggression perpetration and cyber aggression victimization than adolescents from China (perpetration: $M=1.47 ; S D=0.59$; victimization: $M=1.58 ; S D=0.72$ ) and Japan (perpetration: $M=1.19 ; S D=0.26$; victimization: $M=1.26 ; S D=0.41$ ). Cyber aggression involvement was also higher among Chinese adolescents than Japanese adolescents. Boys reported more cyber aggression involvement than girls in China and India. There were no gender differences in cyber aggression perpetration and victimization among Japanese adolescents.

Table 1. Correlation among all variables for Chinese, Indian, and Japanese adolescents.

\begin{tabular}{|c|c|c|c|c|c|c|c|}
\hline & 1 & 2 & 3 & 4 & 5 & 6 & 7 \\
\hline \multicolumn{8}{|l|}{ 1. IND } \\
\hline \multirow{3}{*}{ 2. COLL } & $0.52 * * * /$ & & & & & & \\
\hline & $0.73 * * * /$ & & & & & & \\
\hline & $0.29 * * *$ & & & & & & \\
\hline \multirow{3}{*}{ 3. PA } & $0.18 * * /$ & $0.44 * * * /$ & & & & & \\
\hline & $0.35 * * * /$ & $0.31 * * * /$ & & & & & \\
\hline & 0.08 & $0.52 * * *$ & & & & & \\
\hline \multirow{3}{*}{ 4. CAP } & $0.03 /$ & $-0.16 * * * /$ & $-0.08 * /$ & & & & \\
\hline & $0.24 * * * /$ & $-0.16 * * * /$ & $-0.23 * * * /$ & & & & \\
\hline & 0.03 & -0.01 & -0.02 & & & & \\
\hline \multirow{3}{*}{ 5. CV } & $0.06 /$ & $-0.08 * /$ & $-0.04 /$ & $0.71 * * * /$ & & & \\
\hline & $0.13 * * /$ & $-0.19 * * * /$ & $-0.24 * * * /$ & $0.67 * * * /$ & & & \\
\hline & 0.09 & -0.03 & -0.05 & $0.52 * * *$ & & & \\
\hline \multirow{3}{*}{ 6. FAP } & $0.06 /$ & $-0.20 * * * /$ & $-0.34 * * * /$ & $0.27 * * * /$ & $0.17 * * * /$ & & \\
\hline & $0.21 * * * /$ & $-0.03 /$ & $-0.20 * * * /$ & $0.11 * /$ & $0.23 * * * /$ & & \\
\hline & 0.01 & $-0.23 * *$ & $-0.37 * * *$ & $0.20 * * *$ & $0.15 * * *$ & & \\
\hline \multirow{3}{*}{ 7. FV } & $0.13 * * * /$ & $-0.20 * * * /$ & $-0.31 * * * /$ & $0.14 * * * /$ & $0.18 * * * /$ & $0.48 * * * /$ & \\
\hline & $0.14 * * * /$ & $-0.09 * /$ & $-0.31 * * * /$ & $0.14 * * /$ & $0.13 * * /$ & $0.31 * * * /$ & --- \\
\hline & 0.01 & $-0.29 * * *$ & $-0.48 * * *$ & $0.25 * * *$ & $0.32 * * *$ & $0.65 * * *$ & \\
\hline
\end{tabular}

Note: $\mathrm{IND}=$ individualism; $\mathrm{COLL}=$ collectivism; PA $=$ peer attachment; $\mathrm{CAP}=$ cyber aggression perpetration; $\mathrm{CV}=$ cyber victimization; $\mathrm{FAP}=$ face-to-face aggression perpetration; $\mathrm{FV}$ = face-to-face victimization. The first number is the correlation for Chinese adolescents. The second number is the correlation for Indian adolescents. The third number is the correlation for Japanese adolescents. ${ }^{*} p<0.05$; $* * p<0.01 ; * * * p<0.001$. 
Table 2. Means and standard deviations of cyber aggression perpetration and victimization for China, India, and Japan.

\begin{tabular}{ccccccc}
\hline Country & \multicolumn{2}{c}{ Cyber Aggression Perpetration } & \multicolumn{3}{c}{ Cyber Victimization } \\
\hline & $\begin{array}{c}\text { Full Sample } M \\
(S D)\end{array}$ & Girls $M(S D)$ & $\begin{array}{c}\text { Boys } M \\
(S D)\end{array}$ & $\begin{array}{c}\text { Full Sample } M \\
(S D)\end{array}$ & Girls $M(S D)$ & Boys $M$ \\
& $1.47(0.59)_{\mathrm{ab}}$ & $1.40(0.43)$ & $1.55(0.61)$ & $1.58(0.72)_{\mathrm{ab}}$ & $1.50(0.67)$ & $1.67(0.79)$ \\
China & $1.86(0.74)_{\mathrm{a}}$ & $1.71(0.63)$ & $1.99(0.78)$ & $1.79(0.86) \mathrm{a}$ & $1.65(0.80)$ & $1.92(0.91)$ \\
India & $1.19(0.26)_{\mathrm{ab}}$ & $1.20(0.26)$ & $1.12(0.24)$ & $1.26(0.41)_{\mathrm{ab}}$ & $1.28(0.42)$ & $1.24(0.41)$ \\
\hline Japan & & & & & & \\
\end{tabular}

Note: Covariates include face-to-face aggression perpetration, face-to-face victimization, individualism and collectivism. Means within a column sharing the same subscript letter were found to be significantly different.

\subsection{Peer Attachment and Cyber Aggression Involvement}

Before conducting the analysis, adolescents were split into the following groups based on the means of cyber aggression perpetration $(M=1.51, S D=0.66)$ and cyber aggression victimization $(M=1.56$, $S D=0.75$ ). The following is the breakdown of the groups: cyberaggressors-cyber victims (Group 1; $n=433$ ), cyberaggressors (Group 2; $n=127$ ), cybervictims (Group 3; $n=150$ ), and the uninvolved (Group 4; $n=866$ ). An ANOVA was conducted with parental attachment as the dependent variable, and country, gender, and group as the independent variables. Face-to-face aggression involvement, individualism, and collectivism were included as covariates. Three two-way interactions were included between country and groups, country and gender, and gender and groups. A three-way interaction was also included among country, gender, and groups. Main effects of gender $(F(1,1565)=32.82$, $p<0.001)$ and groups $(F(3,1565)=14.78, p<0.001)$ were found. The main effect of country and the interactions were not significant. Girls $(M=3.78, S D=0.04)$ reported more peer attachment when compared to boys $(\mathrm{M}=3.45, \mathrm{SD}=0.04)$. Uninvolved adolescents $(M=3.88, S D=0.03)$ had greater peer attachment when compared to the other groups (cyberaggressors-cybervictims: $M=3.30$, $S D=0.05$; cyberaggressors: $M=3.69, S D=0.06$; cybervictims: $M=3.53, S D=0.06$ ). Cybervictims had lower levels of peer attachment than cyberaggressors and uninvolved adolescents. Cyberaggressors-cybervictims had the lowest peer attachment when compared to cybervictims, cyberaggressors, and uninvolved adolescents.

\section{Discussion}

The purposes of this study were twofold. The first aim was to investigate the conjoint influence of country of origin and gender on cyber aggression involvement among Chinese, Indian, and Japanese adolescents. The second aim was to examine the combined effects of country of origin, gender, and the cyber aggression involvement classifications on peer attachment. Results from the present study provide further evidence that cyber aggression is an issue impacting adolescents across the world. The findings of the present study contribute greatly to the body of literature on cyber aggression involvement because cultural values and face-to-face aggression involvement were included as covariates. 
Providing support for one of the study's hypotheses, Indian adolescents reported greater cyber aggression perpetration and victimization than adolescents from either China or Japan. Such findings might be supported by the literature, suggesting that Indian culture promotes and rewards both individualistic and collectivistic behaviors [40]. Given their stronger tendency toward individualism than adolescents in China or Japan, adolescents from India might be more at risk for cyber aggression involvement, which is further supported from the literature linking more face-to-face bullying and victimization among adolescents from individualistic countries (e.g., the United States) than collectivistic countries (e.g., China, Japan) [37-39]. Furthermore, Chinese adolescents reported higher cyber aggression involvement when compared to Japanese adolescents, which supported the study's hypotheses. This finding is difficult to reconcile with the literature, considering that both countries highly value collectivism and that collectivism is usually associated with less bullying involvement [40]. Another possibility is that China's greater internet consumption might indicate that Chinese adolescents also spend more time using the internet than Japanese adolescents. Access to the internet and frequency of usage is a risk factor associated with cyber aggression involvement, which might indicate that Chinese adolescents are more at risk than Japanese adolescents [30,31]. Such findings are also aligned with other work in Japan, revealing that Japanese adolescents rarely reported being involved in cyberbullying [34].

Understanding cyber aggression involvement in Asia is better understood by focusing on country of origin and gender differences, which reveal complex patterns. The significant two-way interaction between country of origin and gender suggests that boys reported more cyber aggression involvement in China and India than girls in these countries. Finding that Chinese boys perpetrated and were victimized by cyber aggression more often than Chinese girls is consistent with the literature on gender differences in Chinese adolescents' involvement in cyberbullying [26,32,39]. The findings from India are difficult to compare with the literature since no research has been conducted on cyber aggression involvement in this country. No gender differences were found for cyber aggression perpetration and victimization among Japanese adolescents. This result is not consistent with the literature. For instance, Barlett et al. [33] found that Japanese males had higher levels of cyberbullying perpetration than Japanese females. One possibility for this inconsistent finding is that Barlett and colleagues' study included young adults, whereas the present study included adolescents. Such differences might reflect developmental differences in the samples.

Concerning peer attachment, country of origin was not significant. Thus, adolescents in China, India, and Japan did not differ in their levels of peer attachment. Such findings might reflect the focus on collectivistic values within their countries, which emphasize interdependence and possibly positive peer relationships [40]. Gender was significant, indicating that girls reported more peer attachment when compared to boys, no matter their country of origin. This finding is consistent with a recent meta-analysis on gender differences in peer attachment [50]. In addition, uninvolved adolescents reported greater peer attachment when compared to cyberaggressors-cybervictims, cybervictims, and cyberaggressors, which is supported by the literature [53]. Furthermore, cyberaggressors-cybervictims had the worst levels of peer attachment when compared to cybervictims and cyberaggressors. The findings regarding cyber aggression involvement supported the study's hypotheses. Unlike Burton and colleagues [53], the present study also found that cybervictims had lower levels of peer attachment than cyberbullies. However, this finding is supported by the literature on face-to-face bullying 
involvement [59-61]. The interaction among country of origin, gender, and cyber aggression involvement was not significant. This was not expected since it was hypothesized that Indian adolescents' greater involvement in cyber aggression would worsen their peer attachment. Such a finding might suggest that collectivism serves some type of protective function. In their review of the ecological contexts of bullying, Huang and colleagues [37] suggested that the macrosystem, particularly the emphasis on collectivism versus individualism, might mitigate the negative effects associated with face-to-face bullying involvement among Chinese children and adolescents.

\section{Limitations and Future Directions}

Even though the present study provided much needed information concerning cyber aggression perpetration and victimization in China, India, and Japan, there are a few limitations that should be noted and addressed in future research. First, this study relied on self-reports to assess face-to-face and cyber aggression perpetration and victimization. A multiple informant approach is needed in this research as it reduces the biases associated with self-reports. In addition, recent research has demonstrated the strength of utilizing peer-nominations to assess peer-based cyber aggression involvement [2,11]. Second, this study utilized a concurrent research design to assess cyber aggression perpetration and victimization. Thus, it is impossible to understand the temporal ordering of peer attachment and cyber aggression involvement, and future research should focus on utilizing longitudinal designs.

\section{Conclusions}

The present study provided a much needed examination of the differences in cyber aggression perpetration and victimization among Chinese, Indian, and Japanese adolescents as well as the differences in the cyber aggression involvement classifications for peer attachment. It is also among a few studies to control for face-to-face aggression involvement and cultural values when examining these differences, which is a methodological improvement and an important direction for researchers interested in the role of culture in cyber aggression perpetration and victimization. Despite the differences found in the study, these findings suggest that more research should be conducted on cyber aggression involvement among adolescents in China, India, and Japan. This is incredibly important for cyber aggression perpetration and victimization in India as Indian adolescents had the highest levels of these behaviors and victimization when compared to Chinese and Japanese adolescents. This study may inform school personnel in these countries concerned with identifying risk factors associated with adolescents' cyber aggression involvement based on their gender and their levels of peer attachment.

\section{Acknowledgments}

This work was supported by the project "Employment of Best Young Scientists for International Cooperation Empowerment" (CZ.1.07/2.3.00/30.0037) co-financed from European Social Fund and the state budget of the Czech Republic. This work was also partially supported by JSPS KAKENHI Grant-in-Aid for Young Scientists (B) Grant Number 26870535. 


\section{Author Contributions}

Michelle F. Wright developed the study proposal, analyzed data, and wrote and edited the manuscript. Ikuko Aoyama coordinated data collection, collected data, and edited the manuscript. Shanmukh V. Kamble coordinated data collection, collected data, and edited the manuscript. Zheng Li coordinated data collection, collected data, and edited the manuscript. Shruti Soudi coordinated data collection, and collected data. Li Lei coordinated data collection. Chang Shu coordinated data collection.

\section{Conflicts of Interest}

The authors declare no conflict of interest.

\section{References}

1. Ybarra, M.L.; Diener-West, M.; Leaf, P. Examining the overlap in internet harassment and school bullying: Implications for school intervention. J. Adolesc. Health 2007, 1, S42-S50.

2. Wright, M.F.; Li, Y. The association between cyber victimization and subsequent cyber aggression: The moderating effect of peer rejection. J. Youth Adolesc. 2013, 42, 662-674, doi:10.1007/s10964-012-9903-3.

3. Grigg, D.W. Cyber-aggression: Definition and concept of cyberbullying. Aust. J. Guid. Counsell. 2010, 20, 143-156, doi:10.1375/ajgc.20.2.143.

4. Bauman, S. Cyberbullying in a rural intermediate school: An exploratory study. J. Early Adolesc. 2010, 30, 803-833, doi:10.1177/0272431609350927.

5. Patchin, J.W.; Hinduja, S. Traditional and nontraditional bullying among youth: A test of general strain theory. Youth Soc. 2011, 43, 727-775, doi:10.1177/0044118X10366951.

6. Pornari, C.D.; Wood, J. Peer and cyber aggression in secondary school students: The role of moral disengagement, hostile attribution bias, and outcome expectancies. Aggress. Behav. 2010, 36, 81-94, doi;10.1002/ab.20336.

7. Sontag, L.M.; Clemans, K.H.; Graber, J.A.; Lyndon, S. Traditional and cyber aggressors and victims: A comparison of psychosocial characteristics. J. Youth Adolesc. 2011, 40, 392-404, doi:10.1007/s10964-010-9575-9.

8. Topcu, C.; Erdur-Baker, O. Affective and cognitive empathy as mediators of gender differences in cyber and traditional bullying. Sch. Psychol. Int. 2012, 33, 550-561, doi:10.1177/0143034312446882.

9. Ang, R.P.; Goh, D.H. Cyberbullying among adolescents: The role of affective and cognitive empathy, and gender. Child Psychiatry Hum. Dev. 2010, 41, 387-397, doi:10.1007/s10578-010-0176-3.

10. Fanti, K.A.; Demetriou, A.G.; Hawa, V.V. A longitudinal study of cyberbullying: Examining risk and protective factors. Eur. J. Dev. Psychol. 2012, 9, 168-181, doi:10.1080/17405629.2011.643169.

11. Wright, M.F. Longitudinal investigation of the associations between adolescents' popularity and cyber social behaviors. J. Sch. Violence 2014, 13, 291-314, doi:10.1080/15388220.2013.849201.

12. Campbell, M.; Spears, B.; Slee, P.; Bulter, D.; Kift, S. Victims' perceptions of traditional and cyberbullying and the psychosocial correlates of their victimization. Emot. Behav. Diffic. 2012, 17, 389-401, doi:10.1080/13632752.2012.704316.

13. Kowalski, R.M.; Limber, S.P. Psychological, physical, and academic correlates of cyberbullying and traditional bullying. J. Adolesc. Health 2013, 53, S13-S20, doi:10.1016/j.jadohealth.2012.09.018. 
14. Schenk, A.M.; Fremouw, W.J.; Keelan, C.M. Characteristics of college cyberbullies. Comput. Hum. Behav. 2013, 29, 2320-2327, doi:10.1016/j.chb.2013.05.013.

15. Beran, T.; Li, Q. The relationship between cyberbullying and school bullying. J. Stud. Wellbeing 2007, 1, 15-33.

16. Huang, Y.; Chou, C. An analysis of multiple factors of cyberbullying among junior high school students in Taiwan. Comput. Hum. Behav. 2010, 26, 1581-1590, doi:10.1016/j.chb.2010.06.005.

17. Katzer, C.; Fetchenhauer, D.; Belschak, F. Cyberbullying in chatrooms: Who are the victims? J. Media Psychol. 2009, 21, 25-36, doi:10.1027/1864-1105.21.1.25.

18. Heirman, W.; Walrave, M. Predicting adolescent perpetration in cyberbullying: An application of the theory of planned behavior. Psicothema 2012, 24, 614-620.

19. Festl, R.; Schwarkow, M.; Quandt, T. Peer influence, internet use and cyberbullying: A comparison of different context effects among German adolescents. J. Child. Media 2013, 7, 446-462.

20. Corcoran, L.; Connolly, I.; O’Moore, M. Cyberbullying in Irish schools: An investigation of personality and self-concept. Ir. J. Psychol. 2012, 33, 153-165.

21. Brighi, A.; Guarini, A.; Melotti, G.; Galli, S.; Genta, M.L. Predictors of victimisation across direct bullying, indirect bullying and cyberbullying. Emot. Behav. Diffic. 2012, 17, 375-388, doi:10.1080/13632752.2012.704684.

22. Gamez-Guadix, M.; Orue, I.; Smith, P.K.; Calvete, E. Longitudinal and reciprocal relations of cyberbullying with depression, substance use, and problematic internet use among adolescents. J. Adolesc. Health 2013, 53, 446-452, doi:10.1016/j.jadohealth.2013.03.030.

23. Beckman, L.; Hagquist, C.; Hellstrom, L. Does the association with psychosomatic health problems differ between cyberbullying and traditional bullying? Emot. Behav. Diffic. 2012, 17, 421-434, doi:10.1080/13632752.2012.704228.

24. Laftman, S.B.; Modin, B.; Ostberg, V. Cyberbullying and subjective health: A large-scale study of students in Stockholm, Sweden. Child. Youth Serv. Rev. 2013, 35, 112-119, doi:10.1016/j.childyouth.2012.10.020.

25. Erdur-Baker, O. Cyberbullying and its correlation to traditional bullying, gender and frequent and risky usage of internet-mediated communication tools. New Media Soc. 2010, 12, 109-125, doi:10.1177/1461444809341260.

26. Zhou, Z.; Tang, H.; Tian, Y.; Wei, H.; Zhang, F.; Morrison, C.M. Cyberbullying and its risk factors among Chinese high school students. Sch. Psychol. Int. 2013, 34, 630-647, doi:10.1177/0143034313479692.

27. Jang, H.; Song, J.; Kim, R. Does the offline bully-victimization influence cyberbullying behavior among youths? Application of general strain theory. Comput. Hum. Behav. 2014, 31, 85-93, doi:10.1016/j.chb.2013.10.007.

28. Kwan, G.C.E.; Skoric, M.M. Facebook bullying: An extension of battles at school. Comput. Hum. Behav. 2013, 29, 16-25, doi:10.1016/j.chb.2012.07.014.

29. Internet Live Stats. Available online: http://www.internetlivestats.com/ (accessed on 15 November 2014).

30. Livingstone, S.; Smith, P.K. Annual research review: Harms experienced by child users of online and mobile technologies: The nature, prevalence and management of sexual and aggressive risks in the digital age. J. Child Psychol. Psychiatry 2014, 55, 635-654, doi:10.1111/jcpp.12197. 
31. Park, S.; Na, E.; Kim, E. The relationship between online activities, netiquette and cyberbullying. Child. Youth Serv. Rev. 2014, 42, 74-81, doi:10.1016/j.childyouth.2014.04.002.

32. Li, J.; Gao, X.; Shi, G. Characteristics of cyberbullying among adolescents. Chin. Ment. Health J. 2013, 27, 43-48.

33. Barlett, C.P.; Gentile, D.A.; Anderson, C.A.; Suzuki, K.; Sakamoto, A.; Yamaoka, A.; Katsura, R. Cross-cultural differences in cyberbullying behavior: A short-term longitudinal study. J. Cross Cult. Psychol. 2013, 45, 300-313, doi:10.1177/0022022113504622.

34. Strohmeier, D.; Aoyama, I.; Gradinger, P.; Toda, Y. Cybervictimization and cyberaggression in Eastern and Western countries: Challenges of constructing a cross-cultural appropriate scale. In Principles of Cyberbullying Research: Definitions, Measures, and Methodology; Bauman, S., Cross, D., Walker, J.L., Eds.; Routledge: New York, NY, USA, 2013; pp. 202-221.

35. Halder, D.; Jaishankar, K. Cyber gender harassment and secondary victimization: A comparative analysis of the United States, the UK, and India. Vict. Offenders 2011, 6, 386-398, doi:10.1080/15564886.2011.607402.

36. Nickerson, A.; Nagle, R. Parent and peer attachment in late childhood and early adolescence. J. Early Adolesc. 2005, 25, 223-249, doi:10.1177/0272431604274174.

37. Huang, H.; Hong, J.S.; Espelage, D.L. Understanding factors associated with bullying and peer victimization in Chinese schools within ecological contexts. J. Child Fam. Stud. 2013, 22, 881-892, doi:10.1007/s10826-012-9647-4.

38. Menzer, M.M.; Torney-Purta, J. Individualism and socioeconomic diversity at school as related to perceptions of the frequency of peer aggression in fifteen countries. J. Adolesc. 2012, 35, 1285-1294, doi:10.1016/j.adolescence.2012.04.013.

39. Nesdale, D.; Naito, M. Individualism-collectivism and the attitudes to school bullying of Japanese and Australian Students. J. Cross Cult. Psychol. 2005, 36, 537-556, doi:10.1177/0022022105278541.

40. Singelis, T.M. The measurement of independent and interdependent self-construals. Personal. Soc. Psychol. Bull. 1994, 20, 580-591, doi:10.1177/0146167294205014.

41. Bauman, S.; Toomey, R.B.; Walker, J.L. Associations among bullying, cyberbullying, and suicide in high school students. J. Adolesc. 2013, 36, 341-350, doi:10.1016/j.adolescence.2012.12.001.

42. Gradinger, P.; Strohmeier, D.; Spiel, C. Traditional bullying and cyberbullying. J. Psychol. 2009, $217,205-213$.

43. Hinduja, S.; Patchin, J.W. Cyberbullying: An exploratory analysis of factors related to offending and victimization. Deviant Behav. 2008, 29, 129-156, doi:10.1080/01639620701457816.

44. Hu, Y.; Fan, C.; Zhang, F.; Zhou, R. Behavioral characteristics of different roles in cyberbullying and relation to depression in junior students. Chin. Ment. Health J. 2013, 27, 913-917.

45. Juvonen, J.; Graham, S.; Schuster, M. Bullying among young adolescents: The strong, the weak, and the troubled. Pediatrics 2003, 112, 1231-1237.

46. DeMonchy, M.; Pijl, S.; Zandberg, T. Discrepancies in judging social inclusion and bullying of pupils with behaviour problems. Eur. J. Spec. Needs Educ. 2004, 19, 317-330, doi:10.1080/0885625042000262488.

47. Leary, M.; Twenge, J.; Quinlivan, E. Interpersonal rejection as a determinant of anger and aggression. Personal. Soc. Psychol. Rev. 2006, 10, 111-132, doi:10.1207/s15327957pspr1002_2.

48. Laible, D.; Carlo, G.; Raffaelli, M. The differential relations of parent and peer attachment to adolescent adjustment. J. Youth Adolesc. 2000, 29, 45-59, doi:10.1023/A:1005169004882. 
49. Burton, K.A.; Florell, D.; Wygant, D.B. The role of peer attachment and normative beliefs about aggression on traditional bullying and cyberbullying. Psychol. Sch. 2013, 50, 103-114, doi:10.1002/pits.21663.

50. Gorrese, A.; Ruggieri, R. Peer attachment: A meta-analytic review of gender and age differences and associations with parent attachment. J. Youth Adolesc. 2012, 41, 650-672, doi:10.1007/s10964-012-9759-6.

51. Matsumoto, D; Juang, L. Culture and Psychology; Wadsworth: Belmont, CA, USA, 2004.

52. Pearson, J.C.; Child, J.T. A cross-cultural comparison of parental and peer attachment styles among adult children from the United States, Puerto Rico, and India. J. Intercult. Commun. Res. 2007, 36, 15-32.

53. Laghi, F.; Pallini, S.; Baiocco, R.; Dimitrova, R. Parent and peer attachment and psychosocial adjustment in Chinese immigrant adolescents in Italy. Adv. Immigr. Fam. Res. 2014, 1, 259-273.

54. Yang, H.; Cai, T.; He, Y. Parent attachment, peers attachment and high school students' behavior problems. Chin. J. Clin. Psychol. 2010, 18, 107-108.

55. Li, Y.; Wang, M.; Wang, C.; Shi, J. Individualism, collectivism, and Chinese adolescents' aggression: Intracultural variations. Aggress. Behav. 2010, 36, 187-194, doi:10.1002/ab.20341.

56. Triandis, H.C.; Gelfand, M.J. Converging measurement of horizontal and verticial individualism and collectivism. J. Personal. Soc. Psychol. 1998, 74, 118-128, doi:10.1037/0022-3514.74.1.118.

57. Wright, M.F.; Li, Y.; Shi, J. Chinese adolescents' social status goals: Associations with behaviors and attributions for relational aggression. Youth Soc. 2012, 46, 566-588, doi:10.1177/0044118X12448800.

58. Armsden, G.C.; Greenberg, M.T. The Inventory of Parent and Peer Attachment: Relationships to well-being in adolescence. J. Youth Adolesc. 1987, 16, 427-454, doi:10.1007/BF02202939.

59. Marini, Z.A.; Dane, A.V.; Bosacki, S.L.; YLC-CURA. Direct and indirect bully-victims: Differential psychosocial risk factors associated with adolescents involve in bullying and victimization. Aggress. Behav. 2006, 32, 551-569, doi:10.1002/ab.20155.

60. Vassallo, S.; Edwards, B.; Renda, J.; Olsson, C.A. Bullying in early adolescence and antisocial behavior and depression six years later: What are the protective factors. J. Sch. Violence 2014, 13, 100-124, doi:10.1080/15388220.2013.840643.

61. Walden, L.M.; Beran, T.N. Attachment quality and bullying behavior in school-aged youth. Can. J. Sch. Psychol. 2010, 25, 5-18, doi:10.1177/0829573509357046.

(C) 2015 by the authors; licensee MDPI, Basel, Switzerland. This article is an open access article distributed under the terms and conditions of the Creative Commons Attribution license (http://creativecommons.org/licenses/by/4.0/). 